\title{
Multilocus fragment analysis of Cryptosporidium parvum from pre-weaned calves in Colombia
}

\author{
Catalina Avendaño ${ }^{\mathrm{a}}$, Ana Ramo ${ }^{\mathrm{b}}$, Claudia Vergara-Castiblanco ${ }^{\mathrm{b}}$, Luis V. Monteagudo ${ }^{\mathrm{c}, \mathrm{d}}$, \\ Caridad Sánchez-Acedo ${ }^{\mathrm{b}}$, Joaquín Quílez ${ }^{\mathrm{b}, \mathrm{d}, *}$ \\ ${ }^{a}$ Faculty of Animal Sciences, Universidad de Ciencias Aplicadas y Ambientales, U.D.C.A, Calle 222 \# 55 - 37, Bogotá, Colombia \\ ${ }^{\mathrm{b}}$ Department of Animal Pathology, Faculty of Veterinary Sciences, University of Zaragoza, Miguel Servet 177, 50013, Zaragoza, Spain \\ ${ }^{\mathrm{c}}$ Department of Anatomy, Embryology and Genetics, Faculty of Veterinary Sciences, University of Zaragoza, Miguel Servet 177, 50013, Zaragoza, Spain \\ ${ }^{\mathrm{d}}$ Agrifood Institute of Aragon (IA2), University of Zaragoza-CITA, Miguel Servet 177, 50013, Zaragoza, Spain
}

\section{A R T I C L E I N F O}

\section{Keywords:}

Cryptosporidium parvum

Dairy calves

Microsatellites

Minisatellites

gp60 subtypes

Colombia

\begin{abstract}
A B S T R A C T
The intra-species genetic diversity of Cryptosporidium parvum in dairy cattle farms in the central area of Colombia was investigated using a multilocus fragment typing approach with nine variable-number tandem-repeat (VNTR) loci and the gp60 gene. Genomic DNA of $70 \mathrm{C}$. parvum isolates from pre-weaned calves in 32 farms was analysed. Most markers showed two (ML1, MSB, CP47, and MSC6-7) or three alleles (5B12, Cgd2_3850, and Cgd6_5400), although they exhibited a major allele accounting for more than $69 \%$ of specimens, which explains their low discriminatory index. The TP14 microsatellite was monomorphic while a total of six alleles were found at the ML2 microsatellite. The two novel allelic variants (219bp, 245bp) exhibited by more than $36 \%$ of specimens at the latter locus were a remarkable finding. The 10-markers typing tool provided a Hunter-Gaston discriminatory value of 0.940 (95\% CI, $0.918-0.961$ ) and differentiated 22 multilocus subtypes (MLTs). Nevertheless, the combination of the three most informative markers (ML2, gp60, and Cgd2_3850) differentiated 68\% of MLTs and hardly impaired the discriminatory index. The fact that many MLTs (13/22) were distinctive for individual farms provides evidence for the endemic nature of the infection and the major role played by transmission within farms. The eBURST algorithm suggested a low degree of genetic divergence. All but three MLTs were clustered in a clonal complex with a star-like topology typical of clonal expansion, however linkage analysis did not find evidence of linkage disequilibrium. Bayesian analysis also identified a genetic structure with $K=3$ being the best estimation of ancestral clusters, although a large proportion of isolates (35\%) could not be allocated to a single population, which indicates their mixed origin. The results confirm the genetic distinctiveness of $C$. parvum in cattle farms in this geographical area. This is the first multilocus analysis on the intra-specific variability of Cryptosporidium from calves in South America.
\end{abstract}

\section{Introduction}

Molecular tools are essential in unravelling the identity and transmission dynamics of Cryptosporidium isolates circulating in human and animal populations. This protozoan is a major contributor in diarrhoeal disease in humans and livestock worldwide, particularly cattle (Khan et al., 2018). Thirty-one species have been reported so far, based on sequencing of the small-subunit (SSU) rRNA gene which is considered the most reliable locus for identification of Cryptosporidium spp. Two species are responsible for the majority of human infections, including the anthroponotic C. hominis and the zoonotic C. parvum (Ryan et al., 2016). The latter is also one of the most prevalent enteric pathogens associated with neonatal calf diarrhoea, which highlights the economic significance and public health impact of cryptosporidiosis in cattle farms (Thomson et al., 2017).

Subtyping studies have showed a remarkable genetic diversity within $C$. parvum isolates, with the presence of human-specific, animalspecific and zoonotic subtypes. Fourteen allelic families (IIa $\rightarrow$ IIo) have been identified by phylogenetic analysis of the 60-KDa glycoprotein (gp60) gene, as well as several subtypes within each family. Some families, notably IIc, have only been identified in human cases, but others such as IId and especially IIa, are usually identified in both human and animal infections; remarkably, there is a high prevalence of subtype IIaA15G2R1 in humans and cattle in Europe, North America and

\footnotetext{
*Corresponding author at: Department of Animal Pathology, Faculty of Veterinary Sciences, University of Zaragoza, Miguel Servet 177, 50013, Zaragoza, Spain. E-mail address: jquilez@unizar.es (J. Quílez).
} 
Table 1

Primers and conditions of the PCR reactions for the amplification of diverse microsatellites and minisatellites.

\begin{tabular}{|c|c|c|c|c|c|}
\hline Locus & $\begin{array}{l}\text { GenBank } \\
\text { Acces no. }\end{array}$ & Primer sequence $\left(5^{\prime} \rightarrow 3^{\prime}\right)$ & $\begin{array}{l}\text { Annealing temp } \\
\left({ }^{\circ} \mathrm{C}\right)\end{array}$ & Fragment size range (bp) & Reference \\
\hline \multirow[t]{3}{*}{ ML1 } & \multirow[t]{3}{*}{ G35348 } & F1: CATGAGCTAAAAATGGTGG & 55 & \multirow[t]{3}{*}{$218-242$} & \multirow[t]{3}{*}{ (Cacciò et al., 2000; Chalmers et al., 2005) } \\
\hline & & F2: CTAAAAATGGTGGAGAATATTC & & & \\
\hline & & R: HEX-CAACAAAATCTATATCCTC & 50 & & \\
\hline \multirow[t]{2}{*}{ ML2 } & \multirow[t]{2}{*}{ AF344880 } & F: CAATGTAAGTTTACTTATGATTAT & 50 & \multirow[t]{2}{*}{$180-237$} & \multirow[t]{2}{*}{ (Cacciò et al., 2001) } \\
\hline & & R: FAM-CGACTATAAAGATGAGAGAAG & & & \\
\hline \multirow{4}{*}{ TP14 } & \multirow[t]{4}{*}{ XM627041 } & F1: TAATGCCCACCCATCTTCTT & 61 & \multirow{4}{*}{$279-333$} & \multirow{4}{*}{ (Mallon et al., 2003; Quílez et al., 2011) } \\
\hline & & R1: TCCATCTGGGTCCATTTAGC & & & \\
\hline & & F2: CTAACGTTCACAGCCAACAGTACC & 62 & & \\
\hline & & R2: FAM-GTACAGCTCCTGTTCCTGTTG & & & \\
\hline \multirow[t]{2}{*}{$5 B 12$} & \multirow[t]{2}{*}{ AQ449854 } & F: TGACGATGAAGATGAGGGAAC & 60 & \multirow[t]{2}{*}{ 134-155 } & \multirow[t]{2}{*}{ (Quílez et al., 2011) } \\
\hline & & R: HEX-CAGGACAGATTTAGGAGGAGGA & & & \\
\hline \multirow[t]{4}{*}{ CP47 } & \multirow{4}{*}{ AF384127 } & F1: GCTTAGATTCTGATATGGATCTAT & 43 & \multirow[t]{4}{*}{$417-479$} & \multirow[t]{4}{*}{ (Gatei et al., 2007) } \\
\hline & & R1: AGCTTACTGGTCCTGTATCAGTT & 55 & & \\
\hline & & F2: ACCCCAGAAGGCGGACCAAGGTT & & & \\
\hline & & R2: FAM-GTATCGTGGCGTTCTGAATTATCAA & & & \\
\hline \multirow[t]{2}{*}{ MSB } & \multirow[t]{2}{*}{ XM627997 } & F: GGGAGGCATAGGGATGA & 59 & \multirow[t]{2}{*}{$246-324$} & \multirow[t]{2}{*}{ (Tanriverdi and Widmer, 2006) } \\
\hline & & R: TAMRA-CTTTTGATCGCTTCTTTTCCA & & & \\
\hline \multirow[t]{4}{*}{ MSC6-7 } & \multirow[t]{4}{*}{ BX538350 } & F1: ATTGAACAAACGCCGCAAATGTACA & 55 & \multirow[t]{4}{*}{$517-570$} & \multirow[t]{4}{*}{ (Gatei et al., 2007) } \\
\hline & & R1:CGATTATCTCAATATTGGCTGTTATTGC & 55 & & \\
\hline & & F2: GCTATTTGCTATCGTCTCACATAACT & & & \\
\hline & & R2: TAMRA-CTACTGAATCTGATCTTGCATCAAGT & & & \\
\hline \multirow[t]{4}{*}{$\operatorname{cgd2\_ 3850}$} & \multirow[t]{4}{*}{ XM626569 } & F1: ATTGAAGATTGCGGATGATGGGGTT & 70 & \multirow[t]{4}{*}{$151-205$} & \multirow[t]{4}{*}{ (Ramo et al., 2016a). } \\
\hline & & R1: TGGAGCGCCAAGTGCTGAAGA & & & \\
\hline & & F2: ATTTGCTGTTGCAACTGGTG & 61 & & \\
\hline & & R2: TAMRA-GCCAAGTGCTGAAGAAGAGG & & & \\
\hline cgd6_5400 & XM627858 & F: TAATCTTTGCGTGGGACCTC & 60 & $251-312$ & (Ramo et al., 2016a). \\
\hline & & R1: GTGACTTGAATGACCCAGGA & & & \\
\hline & & R2: HEX-TGGAGTTTCTGAGACACAAAGA & 59 & & \\
\hline
\end{tabular}

Australia (Ryan et al., 2014). Another class of highly polymorphic genetic markers characterized by allelic variability in repeat length are microsatellites and minisatellites, also known as short variable-number tandem-repeat (VNTR) loci. These markers are being increasingly used in multilocus schemes to investigate the intra-species diversity of Cryptosporidium spp. Multilocus methods improve resolution over analysis using a single locus, which underestimates genetic diversity where sexual reproduction occurs (Robinson and Chalmers, 2012). Nevertheless, a standardised multilocus scheme is not currently available (Chalmers et al., 2018).

Cryptosporidium has been recognised as a cause of diarrhoea in suckling calves in some South American countries (Del Coco et al., 2008; Meireles, 2010; Díaz-Lee et al., 2011; Pulido-Medellín et al., 2014; Holsback et al., 2018). However, the impact of bovine cryptosporidiosis in this continent is not well documented and data on the genetic diversity of $C$. parvum are limited. Modest numbers of specimens from calves have been characterised by gp60 sequencing in Brazil, Argentina or Chile (Meireles et al., 2011; Paz e Silva et al., 2013; Tomazic et al., 2013; Del Coco et al., 2014; do Couto et al., 2014; Heckler et al., 2015; Mercado et al., 2015; Toledo et al., 2017) and no previous work with VNTR markers has so far been conducted. In Colombia, the single genotyping study reporting the distribution of Cryptosporidium species and gp60 subtypes among pre-weaned calves highlighted this protozoan as a common and widespread pathogen in the central area of the country (Avendaño et al., 2018). Moreover, the latter study revealed the genetic distinctiveness of $C$. parvum in this geographical area, with the presence of eight subtypes within the IIa family, but the overwhelming predominance of an unusual subtype (IIaA18G5R1) previously unreported in natural infections in human or animal hosts. The genetic polymorphisms of $C$. parvum isolates from the latter contribution have been further characterised in the current study. For this purpose, a panel of nine VNTR markers was characterised by fragment length analysis, and the results were combined with the gp60 subtype. Multilocus subtypes were also used to explore the population structure of $C$. parvum in cattle farms in this area of Colombia.

\section{Materials and methods}

\subsection{Cryptosporidium isolates}

Genomic DNA of 70 C. parvum isolates from a previous study was used in this molecular analysis (Avendaño et al., 2018). These isolates were collected between 2010 and 2012 from naturally infected diarrhoeic (n: 25) and non-diarrhoeic (n: 45) calves younger than 35 days. The calves were from 32 dairy cattle farms located in four Departments in the central area of Colombia: Antioquia (n: 1), Boyacá (n: 4), Cundinamarca (n: 26) and Meta (n: 1). One isolate from each of 16 farms and 2 to 6 isolates from each of the remaining 16 farms were used. Calves were maintained under semi-extensive feeding conditions. Cryptosporidium species and C. parvum gp60 subtypes in the previous study were determined based on a PCR-restriction fragment length polymorphism (RFLP) and sequence analyses of the SSU-rRNA and gp60 genes, respectively (Xiao et al., 2001; Alves et al., 2003; Feng et al., 2007).

\subsection{Multilocus fragment typing}

An automated capillary electrophoresis (CE)-based DNA fragment analysis tool was used to categorize each isolate at nine VNTR markers, including five microsatellite (ML1, ML2, TP14, 5B12, CP47) and four minisatellite (MSB, MSC6-7, cgd2_3850, cgd6_5400) loci. The fragments were amplified using single (ML2, 5B12, MSB), heminested (ML1, cgd6_5400) and nested (TP14, CP47, MSC6-7, cgd2_3850) PCRs, with primers and conditions previously described (Gatei et al., 2007; Quílez et al., 2011; Ramo et al., 2016a). Reverse primers labelled with HEX $\left(4,7,2^{\prime}, 4^{\prime}, 5^{\prime}, 7^{\prime}\right.$-hexachloro-6-carboxyfluorescein), FAM (6-carboxyfluorescein), or TAMRA (6-carboxytetra-methylrhodamine) were used to allocate alleles with overlapping peaks to a specific locus. The primers used for PCR analysis of all gene targets, the annealing temperatures used, and the sizes of the expected PCR products are listed in Table 1. 
According to the amplicon intensity, 0.5-to- $2 \mu$ samples of the micro- and minisatellite-labelled PCR products for each C. parvum isolate were mixed and subjected to $\mathrm{CE}$ on a 3500xL Genetic Analyzer and sized automatically using the GeneScan $600 \mathrm{Liz}$ Size Standard (Applied Biosystems, Life Technologies). Data were stored and analysed with the aid of Gene Mapper software (version 4.1) to determine fragment sizes. At least two representative isolates for each allele were amplified using unlabelled primers and the above-mentioned PCR conditions, and they were subsequently analysed by bidirectional sequencing for length confirmation. Allele nomenclature was based on the fragment size (in base pairs) adjusted after comparison with sequence analysis of these representative isolates. Alleles were translated into numbers for multilocus analyses.

\subsection{Multilocus subtype identification}

The alleles at all nine VNTR loci were combined with the gp60 subtype to provide the multilocus subtype (MLT) for each isolate. Each MLT was then assigned a number. The identification of two alleles at a single locus was designated a mixed infection, and the two possible MLTs were considered. The Hunter-Gaston discriminatory index (HGDI) and 95\% confidence intervals were calculated for each VNTR locus and the multilocus analysis. For this purpose, the VNTR diversity and confidence extractor software (V-DICE) available at the Health Protection agency bioinformatics tools website were used (http://www.hpabioinfotools.org.uk/cgi-bin/DICI/DICI.pl) (Hunter and Gaston, 1988).

\subsection{Data analysis}

The evolutionary relationships of the isolates was analysed by the eBURST algorithm (http://eburst.mlst.net/). Clonal complexes were defined as clusters of closely related MLTs that differ from one another at one locus [single locus variants (SLVs)]. The MLTs that were not clustered in any clonal complex were classified as singletons (Feil et al., 2004). Allelic linkage disequilibrium (LD) among different loci was assessed by measuring the standardised index of association $\left(I_{\mathrm{A}} \mathrm{S}\right)$ using the software LIAN v. 3.7 (http://guanine.evolbio.mpg.de/cgi-bin/lian/ lian.cgi.pl) (Haubold and Hudson, 2000). The population structure was determined using the software STRUCTURE v. 2.3 (http://pritchardlab. stanford.edu/structure.html). This program utilises a Bayesian algorithm in order to identify distinct sub-populations and determine fractions of the MLT for each isolate belonging to each subpopulation (Pritchard et al., 2000). The most probable number of ancestral populations was defined by the $K$ value in accordance with previous reports (Evanno et al., 2005).

\subsection{Nucleotide sequence accession numbers}

Representative nucleotide sequences generated in the current study were deposited in the GenBank database under accession numbers MG924432 to MG924435, MG924439 to MG924446 and MG924450.

\section{Results}

\subsection{Allelic diversity}

The numbers and sizes of alleles and the HGDI values identified at each VNTR locus are summarised in Table 2. The analysis of allelic variation revealed that most loci exhibited two (ML1, MSB, CP47, and MSC6-7) or three (5B12, Cgd2_3850, and Cgd6_5400) alleles and the TP14 microsatellite was monomorphic. A major allele was exhibited by more than $69 \%$ of isolates at all these markers, with a single locus (Cgd2_3850) displaying a HDGI value higher than 0.450 . In contrast, a total of six alleles more evenly distributed were identified at the ML2 microsatellite, which explains the highest HGDI value (0.822) exhibited by this marker. This discriminatory index was higher than that reported
Table 2

Microsatellites and minisatellites alleles identified using capillary electrophoresis in C. parvum isolates from calves in Colombia. The adjusted size after sequencing (base pairs) and number assigned to each allele for multilocus analysis is indicated.

\begin{tabular}{|c|c|c|}
\hline Locus Alelle (bp) (assigned number) & $\mathrm{N}^{\circ}$ of isolates (\%) (n: 70) $)^{\mathrm{a}}$ & $\mathrm{N}^{\circ}$ of farms (n: 32) \\
\hline \multicolumn{3}{|l|}{ ML1 $[\text { HGDI }=0.089(0.000-0.182)]^{\mathrm{b}}$} \\
\hline $226(2)$ & $3(4.6)$ & 3 \\
\hline $238(1)$ & $62(95.4)$ & 24 \\
\hline \multicolumn{3}{|c|}{ ML2 $[$ HGDI $=0.822(0.802-0.842)]$} \\
\hline $219(2)^{d}$ & $11(18)$ & 5 \\
\hline $231(1)$ & $15(24.6)$ & 5 \\
\hline $233(3)$ & $11(18)$ & 6 \\
\hline $235(6)$ & $2(3.3)$ & 2 \\
\hline $237(4)$ & $11(18)$ & 8 \\
\hline $245(5)^{d}$ & $11(18)$ & 4 \\
\hline \multicolumn{3}{|l|}{ TP14 [HGDI $=0.000(0.000-0.101)]$} \\
\hline $324(1)$ & $67(100)$ & 30 \\
\hline \multicolumn{3}{|l|}{ MSB $[$ HGDI $=0.031(0.000-0.089)]$} \\
\hline $316(2)$ & $1(1.5)$ & 1 \\
\hline $322(1)$ & $64(98.5)$ & 28 \\
\hline \multicolumn{3}{|c|}{ CP47 $[\text { HGDI }=0.239(0.119-0.360)]^{\mathrm{c}}$} \\
\hline $417(1)$ & $55(85.9)$ & 25 \\
\hline $420(2)$ & $7(10.9)$ & 5 \\
\hline $417+420$ & $2(3.1)$ & 2 \\
\hline \multicolumn{3}{|c|}{ MSC6-7 $[$ HGDI $=0.058(0.000-0.134)]$} \\
\hline $516(2)$ & $1(1.5)$ & 1 \\
\hline $549(1)$ & 64 (97) & 29 \\
\hline $516+549$ & $1(1.5)$ & 1 \\
\hline \multicolumn{3}{|l|}{$5 \mathrm{~B} 12[\mathrm{HGDI}=0.223(0.098-0.347)]$} \\
\hline $167(2)$ & $7(10.9)$ & 6 \\
\hline 169 (1) & $56(87.5)$ & 24 \\
\hline $173(3)^{d}$ & $1(1.6)$ & 1 \\
\hline \multicolumn{3}{|c|}{ Cgd2_3850 [HGDI $=0.459(0.352-0.566)]$} \\
\hline $169(3)^{d}$ & $4(6.1)$ & 1 \\
\hline $193(1)$ & $46(69.7)$ & 21 \\
\hline $199(2)$ & $15(22.7)$ & 10 \\
\hline $193+199$ & $1(1.5)$ & 1 \\
\hline \multicolumn{3}{|c|}{ Cgd6_5400 [HGDI $=0.190(0.072-0.309)]$} \\
\hline $271(3)$ & $1(1.6)$ & 1 \\
\hline 277 (1) & $56(88.9)$ & 24 \\
\hline $283(2)$ & $1(1.6)$ & 1 \\
\hline $277+283$ & $5(7.9)$ & 1 \\
\hline \multicolumn{3}{|l|}{ GP60 [HGDI $=0.506(0.363-0.650)]$} \\
\hline IIaA15G2R1 (1) & $5(7.6)$ & 3 \\
\hline IIaA16G2R1 (2) & $3(4.5)$ & 1 \\
\hline IIaA17G4R1 (3) & $2(3)$ & 1 \\
\hline IIaA18G5R1 (4) & $46(69.7)$ & 22 \\
\hline IIaA19G6R1 (5) & $2(3)$ & 2 \\
\hline IIaA20G5R1 (6) & $3(4.5)$ & 3 \\
\hline IIaA20G6R1 (7) & $4(6.1)$ & 1 \\
\hline IIaA20G7R1 (8) & $1(1.5)$ & 1 \\
\hline
\end{tabular}

${ }^{\text {a }}$ Not all 70 C. parvum isolates could be typed at every loci.

b Hunter-Gaston Discriminatory Index (index [confidence interval 95\%]).

c CP47 alleles were identified as IA29G10 (417bp) and IIA30G10 (420bp) by sequencing, in accordance with the nomenclature proposed by Gatei et al. (2007).

d Alleles not described previously.

for gp60 sequencing, which differentiated a total of eight subtypes in a previous study with the same stock of samples. Sequencing of representative isolates for each allele revealed novel allelic variants at the ML2 (219 and 245bp), 5B12 (173bp) and Cgd2_3850 (169bp) loci.

\subsection{Multilocus subtypes}

The multilocus subtype (MLT) of each isolate was determined based on the combination of alleles at the nine mini- and microsatellite loci and the gp60 subtype. A total of 61 isolates from 26 farms had complete allele data for all ten loci and 22 MLTs were identified (Table 3). The two most prevalent MLTs (13 and 15) were identified in ten specimens and four farms. Nine isolates showing a biallelic profile at a single locus 
Table 3

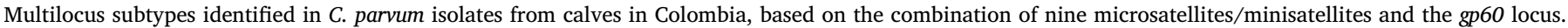

\begin{tabular}{|c|c|c|c|c|c|c|c|c|c|c|c|c|}
\hline \multirow[t]{2}{*}{ MLT } & \multicolumn{10}{|c|}{ Alleles identified at each locus ${ }^{\text {a }}$} & \multirow{2}{*}{$\begin{array}{l}\mathrm{N}^{\mathrm{o}} \text { of isolates } \\
\text { (n: } 61 \text { ) }\end{array}$} & \multirow{2}{*}{$\begin{array}{l}\text { № of farms } \\
\text { (n: 26) }\end{array}$} \\
\hline & TP14 & MSB & MSC 6-7 & ML1 & cgd6_5400 & 5B12 & CP47 & $\operatorname{cgd2} 3850$ & ML2 & GP60 & & \\
\hline 1 & 1 & 1 & 1 & 1 & 1 & 2 & 1 & 2 & 4 & 6 & 3 & 3 \\
\hline 2 & 1 & 1 & 1 & 1 & 1 & 2 & 1 & 2 & 4 & 8 & 1 & 1 \\
\hline 3 & 1 & 1 & 1 & 1 & 1 & 2 & 2 & 1 & 2 & 4 & 3 & 2 \\
\hline 4 & 1 & 1 & 1 & 1 & 1 & 1 & 1 & 1 & 1 & 2 & 3 & 1 \\
\hline 5 & 1 & 1 & 1 & 1 & 1 & 1 & 1 & 1 & 1 & 4 & 3 & 1 \\
\hline 6 & 1 & 1 & 1 & 1 & 1 & 1 & 1 & 1 & 3 & 1 & 3 & 1 \\
\hline 7 & 1 & 1 & 1 & 1 & 1 & 1 & 1 & 1 & 3 & 3 & 2 & 1 \\
\hline 8 & 1 & 1 & 1 & 1 & 1 & 1 & 1 & 1 & 3 & 4 & 4 & 3 \\
\hline 9 & 1 & 1 & 1 & 1 & 1 & 1 & 1 & 1 & 6 & 4 & 1 & 1 \\
\hline 10 & 1 & 1 & 1 & 1 & 1 & 1 & 1 & 1 & 4 & 4 & 5 & 3 \\
\hline 11 & 1 & 1 & 1 & 1 & 1 & 1 & 1 & 1 & 4 & 5 & 2 & 2 \\
\hline 12 & 1 & 1 & 1 & 1 & 1 & 1 & 1 & 1 & 5 & 1 & 1 & 1 \\
\hline 13 & 1 & 1 & 1 & 1 & 1 & 1 & 1 & 1 & 5 & 4 & 10 & 4 \\
\hline 14 & 1 & 1 & 1 & 1 & 1 & 1 & 1 & 1 & 2 & 4 & 2 & 2 \\
\hline 15 & 1 & 1 & 1 & 1 & 1 & 1 & 1 & 2 & 1 & 4 & 10 & 4 \\
\hline 16 & 1 & 1 & 1 & 1 & 1 & 1 & 1 & 3 & 2 & 7 & 4 & 1 \\
\hline 17 & 1 & 1 & 1 & 1 & 1 & 1 & 2 & 1 & 3 & 4 & 1 & 1 \\
\hline 18 & 1 & 1 & 1 & 1 & 1 & 1 & 2 & 1 & 6 & 4 & 1 & 1 \\
\hline 19 & 1 & 1 & 1 & 1 & 1 & 1 & 2 & 1 & 2 & 4 & 4 & 3 \\
\hline 20 & 1 & 1 & 1 & 1 & 2 & 1 & 1 & 1 & 5 & 4 & 5 & 1 \\
\hline 21 & 1 & 1 & 1 & 1 & 3 & 1 & 1 & 1 & 3 & 1 & 1 & 1 \\
\hline 22 & 1 & 1 & 2 & 1 & 1 & 1 & 1 & 1 & 5 & 1 & 1 & 1 \\
\hline
\end{tabular}

a The number assigned to each allele is shown in Table 2.

b Only alleles that amplified at all ten markers were used for the multilocus analysis. Samples revealing mixed infection at a specific marker were unfolded and assigned to the corresponding MLT.

(CP47, cgd6_5400, cgd2_3850 and MSC6-7) were scored as having two potential MLTs; five of these isolates belonged to the same farm and showed a biallelic profile at locus Cgd6_5400. Numerous MLTs (13/22) were distinctive for individual farms. The remaining nine MLTs were simultaneously identified in two or more farms. A comparison between MLTs and the previously reported subtypes determined by gp60 sequencing showed that each subtype corresponded to a single MLT, with the exception of subtypes IIaA15G2R1 and IIaA18G5R1. The multilocus analyses differentiated additional allelic variants in specimens subtyped as IIaA15G2R1 (4 MLTs) and IIaA18G5R1 (12 MLTs). The HGDI value of the ten-satellite tool was 0.940 (95\% CI, $0.918-0.961)$. The inclusion of the three most discriminatory loci (ML2, gp60 and Cgd2_3850) in the multilocus analysis differentiated a total of 15 MLTs and provided a similar value (HGDI: 0.904; 95\% CI, 0.873-0.936).

\subsection{Population analysis}

The eBURST analysis revealed two clonal complexes and one singleton, with the main cluster containing all but three MLTs. The second clonal complex was formed only by two MLTs differing in the gp60 subtype (Fig. 1). The linkage analyses of all specimens as a single population suggested a predominantly panmictic structure within the $C$. parvum population in this geographical area. Evidence of linkage disequilibrium (LE) was not found since the pairwise variance $\left(V_{\mathrm{D}}\right.$ : $1.3196)$ was lower than the $95 \%$ critical value ( $L: 1.3221)$, although the $P$ value almost reached statistical significance $\left(\mathrm{I}_{A} s: 0.0186 ; P: 0.056\right)$. Values remained not significant when counting only once all repeated MLTs $\left(I_{\mathrm{A}} \mathrm{S}: 0.0051 ; V_{\mathrm{D}}: 1.2365 ; L: 1.6017 ; P: 0.378\right)$. The results of analysis with STRUCTURE indicated that the most likely number of clusters was three $(K=3)$. Two thirds of isolates $(65.7 \%)$ were considered to belong to any of the three clusters since the probability of





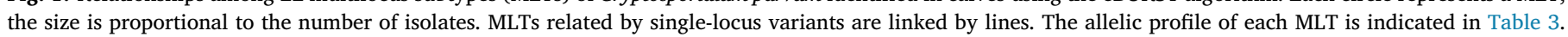



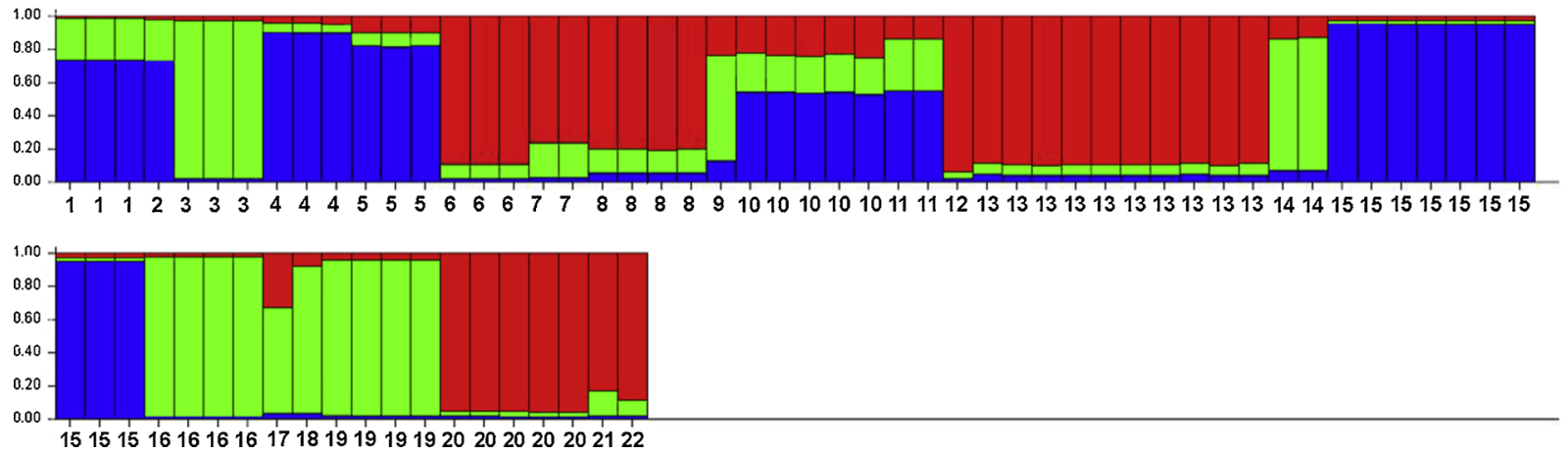



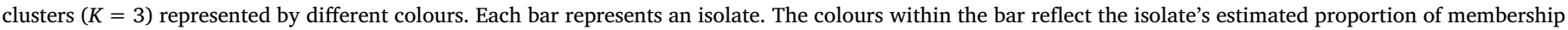

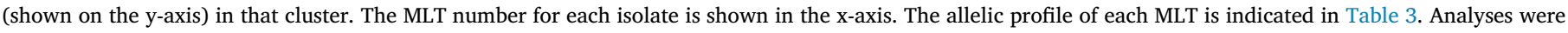
conducted on allelic data at VNTR loci and the GP60 subtype.

belonging to them was higher than 0.8 . The remaining isolates could not be allocated to a single population and were considered to have a mixed ancestry (Fig. 2).

\section{Discussion}

Multilocus genotyping is more informative than the analysis of a single locus in exploring the intra-species diversity of Cryptosporidium, given the occurrence of genetic recombination in the sexual phase of the protozoan life cycle. Combinations with more than 55 VNTR loci have been used to date and recommendations on the validation of markers for a multilocus scheme have been proposed, although no standardised panel has been universally adopted so far (Robinson and Chalmers, 2012; Hotchkiss et al., 2015; Pérez-Cordón et al., 2016; Chalmers et al., 2017, 2018). A review of multilocus studies with $C$. hominis and $C$. parvum revealed that different sets of markers are required for typing each species (Robinson and Chalmers, 2012). Studies with C. parvum from domestic ruminants indicated that differences also apply to host factors, with some loci being much more useful for analysing isolates from either calves or lambs (Ramo et al., 2016a,b).

The genetic diversity or C. parvum in cattle is not well documented in South America. Previous studies based on sequence analysis of the gp60 gene, which is the most popular marker for Cryptosporidium subtyping, have shown the genetic richness of the protozoan in this area. A wide range of subtypes belonging to a single $g p 60$ allelic family have been reported in Brazil (IIaA14G2R1, IIaA14G2R2, IIaA15G2R1, IIaA16G3R2, IIaA17G2R1, IIaA18G1R1, IIaA18G2R2, IIaA19G2R1, IIaA19G2R2, IIaA20G1R1; IIaA20G2R1, IIaA20G2R2) (Meireles et al., 2011; Paz e Silva et al., 2013; do Couto et al., 2014; Heckler et al., 2015; Toledo et al., 2017), Argentina (IIaA16G1R1, IIaA17G1R1, IIaA18G1R1, IIaA19G1R1, IIaA20G1R1, IIaA21G1R1, IIaA22G1R1, IIaA23G1R1) (Tomazic et al., 2013; Del Coco et al., 2014) and Chile (IIaA15G4R1, IIaA16G4R1, IIaA17G4R1) (Mercado et al., 2015). The fact that many of these subtypes were novel allelic variants or were not shared among different countries has been related to the geographic isolation of the protozoan in cattle farms (Avendaño et al., 2018). The latter authors conducted the first large study of Cryptosporidium species and subtypes in Colombia, which revealed the distinct identity of $C$. parvum circulating in calves. A rare subtype, IIaA18G5R1, was by far the most prevalent and widely distributed in the study, and three novel subtypes were found (IIaA19G6R1, IIaA20G6R1 and IIaA20G7R1).

Isolates from the above-mentioned investigation have been further analysed in the present study using a panel of nine VNTR markers, which were selected based on their resolution ability for typing $C$. parvum from calves (Ramo et al., 2016a). None of the VNTR loci exceeded the genetic variability of the $g p 60$ locus ( 8 alleles). Seven markers were either biallelic (ML1, MSB, CP47, MSC6-7) or triallelic (5B12, cgd6_5400, cgd2_3850) but exhibited a major allele accounting for more than $69 \%$ of specimens, which explains their low discriminatory index. It is worth mentioning that no variation was found at the TP14 microsatellite, which is considered a relevant locus in multilocus studies in Europe and the United States (Quílez et al., 2011, 2013; Herges et al., 2012; Hotchkiss et al., 2015). This marker was required to achieve $95 \%$ of MLTs in previous studies with either $C$. parvum or C. hominis (Robinson and Chalmers, 2012). The 324bp fragment exhibited by all isolates in this study was also the predominant allele found in calves in northern Spain (Ramo et al., 2016a). Nevertheless, the latter authors reported two additional allelic variants (333bp and $342 \mathrm{bp}$ ) that increased the HDGI value to 0.663 , which indicates that geographical factors should also be considered when selecting the most informative loci.

The greatest number of alleles was detected at the ML2 locus (6 alleles), which provided a HGDI value even higher than gp60 sequencing. This microsatellite has been reported to be among the most polymorphic markers for typing C. parvum from humans, pre-weaned livestock or waterborne outbreaks in Europe (Cacciò et al., 2001; Hunter et al., 2007, 2008; Quílez et al., 2011, 2013; Díaz et al., 2012). The most prevalent allele at this marker (ML2-231) was also the most common allelic variant in calves in Spain (Quílez et al., 2011; Díaz et al., 2012; Ramo et al., 2016a). Nevertheless, more than $36 \%$ of specimens exhibited two novel allelic variants $219 \mathrm{bp}$ and $245 \mathrm{bp}$ in length, which further supports the genetic uniqueness of $C$. parvum in cattle farms in this geographical area.

The combination of nine VNTR markers with the gp60 subtype provided a more robust analysis than $g p 60$ alone, and identified 22 multilocus subtypes within 61 isolates. Most MLTs (16/22) were differentiated within the two most prevalent subtypes (IIaA15G2R1 and IIaA18G5R1), thereby supporting the usefulness of multilocus typing as compared to sequencing of the gp60 gene. It is significant to note that the combination of only the three most informative markers (ML2, gp60 and Cgd2_3850) differentiated 68\% of MLTs and hardly impaired the discriminatory index, showing a marker redundancy that has been reported in most multilocus schemes (Robinson and Chalmers, 2012). Likewise, the exclusion of $g p 60$ and ML2 loci dramatically reduced the numbers of MLTs $(n=9)$, indicating that both markers contributed the most to the discriminatory power of the multilocus approach.

The genetic diversity found in our study is similar to that reported among 118 calves in the United Kingdom using a six-loci approach (23 MLTs) (Hotchkiss et al., 2015), but much lower to that detected in Ireland (78 MLTs) or Spain (70 MLTs) using panels of seven and twelve markers, respectively (de Waele et al., 2013; Ramo et al., 2016a). Many MLTs (13/22) were confined to individual farms, a finding that has been related to the endemic nature of infection and intensive farming practices with limited exchange of animals between herds (Tanriverdi and Widmer, 2006; Quílez et al., 2011; Drumo et al., 2012; Ramo et al., 2016a). In the current study, dairy calves in most farms (31/32) were 
reared under a semi-extensive system, but the introduction of new animals occurred rarely. Most herds breed their own replacement females rather than buying young heifers, suggesting that Cryptosporidium infection is mainly transmitted within cattle farms.

Analyses of $C$. parvum populations have shown a significant diversity in terms of the role of genetic exchange, with differences at a local geographical level that could determine the degree of recombination (Tanriverdi et al., 2008). Evidence of these variations has been reported in Scotland, with panmictic, epidemic or clonal subpopulations among specimens from humans and livestock (Mallon et al., 2003; Morrison et al., 2008). The C. parvum population structure ranged from basic clonality (humans) to epidemic clonality (livestock) in France, and a predominant pattern of clonality was found in Italy (Ngouanesavanh et al., 2006; Drumo et al., 2012). In contrast, an overall panmitic structure was reported in cattle farms and/or humans in the Upper Midwest of the United States, Ireland and northern Spain (Herges et al., 2012; de Waele et al., 2013; Ramo et al., 2016a).

In the current study, the analysis with the eBURST algorithm showed that all but three MLTs were linked by SLVs, suggesting a low degree of genetic divergence. The distinctive identity of the remaining three MLTs was associated with the gp60 gene, since all these specimens belonged to any of the three subtypes with 20 TCA repeats in the trinucleotide region. MLTs in the main clonal complex formed a network with a star-like structure typical of clonal expansion, but linkage analysis did not find evidence of linkage disequilibrium. Nevertheless, the $P$-value was close to the statistical significance at the $5 \%$ level, advising subsequent studies with more exhaustive sampling. This observation was supported by an analysis using STRUCTURE, which indicated that the population structure is best explicated by $K=3$ ancestral types, but a large proportion of isolates (35\%) could not be assigned to only one of these three populations as they showed mixed ancestry.

To the best of our knowledge, this is the first multilocus analysis on the intra-specific variability of Cryptosporidium from calves in South America. The results reveal that this fragment analysis approach based on the combination of ten markers is a useful tool for strain typing and epidemiological tracking, but should be optimized by selecting the most informative markers at a local level in order to improve both the cost effectiveness and the time involved in testing. Our findings also confirm the distinctiveness of $C$. parvum infection in cattle farms in this geographical area and indicate a moderate genetic diversity. Further investigations with a larger sample set of specimens from this and other areas should be conducted to better understand the genetic diversity of the protozoan in South America.

\section{Declarations of interest}

None.

\section{Acknowledgments}

This study was financially supported by funds from the Government of Aragón and the European Regional Development Fund (A16_17R), the Spanish Ministry of Economy and Competitiveness (AGL201232138) and the Universidad de Ciencias Aplicadas y Ambientales (U.D.C.A) in Bogotá, Colombia. We thank Dr. Demetris Savva (School of Biological Sciences, University of Reading, UK) for a thoughtful and careful review of the manuscript, and Joaquín Medina (Faculty of Veterinary Sciences, University of Zaragoza, Spain) for technical assistance with the Figures.

\section{References}

Alves, M., Xiao, L., Sulaiman, I., Lal, A.A., Matos, O., Antunes, F., 2003. Subgenotype analysis of Cryptosporidium isolates from humans, cattle, and zoo ruminants in Portugal. J. Clin. Microbiol. 41, 2744-2747. https://doi.org/10.1128/JCM.41.6. 2744.
Avendaño, C., Ramo, A., Vergara-Castiblanco, C., Sánchez-Acedo, C., Quílez, J., 2018. Genetic uniqueness of Cryptosporidium parvum from dairy calves in Colombia. Parasitol. Res. 117, 1317-1323. https://doi.org/10.1007/s00436-018-5818-6.

Cacciò, S., Homan, W., Camilli, R., Traldi, G., Kortbeek, T., Pozio, E., 2000. A microsatellite marker reveals population heterogeneity within human and animal genotypes of Cryptosporidium parvum. Parasitology 120, 237-244. https://doi.org/10. 1017/s0031182099005508.

Cacciò, S., Spano, F., Pozio, E., 2001. Large sequence variation at two microsatellite loci among zoonotic (genotype C) isolates of Cryptosporidium parvum. Int. J. Parasitol. 31, 1082-1086. https://doi.org/10.1016/S0020-7519(01)00233-8.

Chalmers, R.M., Ferguson, C., Cacciò, S., Gasser, R.B., Abs EL-Osta, Y.G., Heijnen, L., Xiao, L., Elwin, K., Hadfield, S., Sinclair, M., Stevens, M., 2005. Direct comparison of selected methods for genetic categorisation of Cryptosporidium parvum and Cryptosporidium hominis species. Int. J. Parasitol. 35, 397-410. https://doi.org/10. 1016/j.ijpara.2005.01.001.

Chalmers, R.M., Robinson, G., Hotchkiss, E., Alexander, C., May, S., Gilray, J., Connelly, L., Hadfield, S.J., 2017. Suitability of loci for multiple-locus variable-number of tandem-repeats analysis of Cryptosporidium parvum for inter-laboratory surveillance and outbreak investigations. Parasitology 144, 37-47. https://doi.org/10.1017/ S0031182015001766.

Chalmers, R.M., Pérez-Cordón, G., Cacció, S.M., Klotz, C., Robertson, L.J., 2018. Cryptosporidium genotyping in Europe: the current status and processes for a harmonised multi-locus genotyping scheme. Exp. Parasitol. 191, 25-30. https://doi.org/ 10.1016/j.exppara.2018.06.004.

de Waele, V., Van den Broeck, F., Huyse, T., McGrath, G., Higgins, I., Speybroeck, N., Berzano, M., Raleigh, P., Mulcahy, G.M., Murphya, T.M., 2013. Panmictic structure of the Cryptosporidium parvum population in irish calves: influence of prevalence and host movement. Appl. Environ. Microbiol. 79, 2534-2541. https://doi.org/10.1128/ AEM.03613-12.

Del Coco, V.F., Córdoba, M., Basualdo, J., 2008. Cryptosporidium infection in calves from a rural area of Buenos Aires. Argentina. Vet. Parasitol. 158, 31-35. https://doi.org/10. 1016/j.vetpar.2008.08.018.

Del Coco, V.F., Córdoba, M., Bilbao, G., de Almeida Castro, A.P., Basualdo, J., Fayer, R., Santín, M., 2014. Cryptosporidium parvum GP60 subtypes in dairy cattle from Buenos Aires, Argentina. Res. Vet. Sci. 96, 311-314. https://doi.org/10.1016/j.rvsc.2013.12. 010.

Díaz, P., Hadfield, S.J., Quílez, J., Soilán, M., López, C., Panadero, R., Díez-Baños, P., Morrondo, P., Chalmers, R.M., 2012. Assessment of three methods for multilocus fragment typing of Cryptosporidium parvum from domestic ruminants in north west Spain. Vet. Parasitol. 186, 188-195. https://doi.org/10.1016/j.vetpar.2011.11.039.

Díaz-Lee, A., Mercado, R., Onuoha, E.O., Ozaki, L.S., Muñoz, P., Muñoz, V., Martínez, F.J., Fredes, F., 2011. Cryptosporidium parvum in diarrheic calves detected by microscopy and identified by immunochromatographic and molecular methods. Vet. Parasitol. 176, 139-144. https://doi.org/10.1016/j.vetpar.2010.11.001.

do Couto, M.C., Lima, Mde F., do Bomfim, T.C., 2014. New Cryptosporidium parvum subtypes of IIa subfamily in dairy calves from Brazil. Acta Trop. 130, 117-122. https://doi.org/10.1016/j.actatropica.2013.11.002.

Drumo, R., Widmer, G., Morrison, L.J., Tait, A., Grelloni, V., D’Avino, N., Pozio, E., Cacciò, S.M., 2012. Evidence of host-associated populations of Cryptosporidium parvum in Italy. Appl. Environ. Microbiol. 78, 3523-3529. https://doi.org/10.1128/ AEM.07686-11.

Evanno, G., Regnaut, S., Goudet, J., 2005. Detecting the number of clusters of individuals using the software STRUCTURE: a simulation study. Mol. Ecol. 14, 2611-2620. https://doi.org/10.1111/j.1365-294X.2005.02553.x.

Feil, E.J., Li, B.C., Aanensen, D.M., Hanage, W.P., Spratt, B.G., 2004. eBURST: inferring patterns of evolutionary descent among clusters of related bacterial genotypes from multilocus sequence typing data. J. Bacteriol. 186, 1518-1530. https://doi.org/10. 1128/JB.186.5.1518-1530.2004.

Feng, Y., Ortega, Y., He, G., Das, P., Xu, M., Zhang, X., Fayer, R., Gatei, W., Cama, V. Xiao, L., 2007. Wide geographic distribution of Cryptosporidium bovis and the deerlike genotype in bovines. Vet. Parasitol. 144, 1-9. https://doi.org/10.1016/j.vetpar. 2006.10.001.

Gatei, W., Das, P., Dutta, P., Sen, A., Cama, V., Lal, A.a, Xiao, L., 2007. Multilocus sequence typing and genetic structure of Cryptosporidium hominis from children in Kolkata. India. Infect. Genet. Evol. 7, 197-205. https://doi.org/10.1016/j.meegid 2006.08.006.

Haubold, B., Hudson, R.R., 2000. LIAN 3.0: detecting linkage disequilibrium in multilocus data. Linkage Analysis. Bioinformatics 16, 847-848. https://doi.org/10.1093/ bioinformatics $/ 16.9 .847$.

Heckler, R., Borges, D., Bacha, F., Onizuka, M., Teruya, L., Neves, J., Leal, C., Lemos, R., Meireles, M., Borges, F., 2015. First genetic identification of Cryptosporidium parvum subtype IIaA14G2R1 in beef cattle in Brazil. Prev. Vet. Med. 121, 391-394. https:// doi.org/10.1016/j.prevetmed.2015.08.016.

Herges, G.R., Widmer, G., Clark, M.E., Khan, E., Giddings, C.W., Brewer, M., Mcevoy, J.M., 2012. Evidence that Cryptosporidium parvum populations are panmictic and unstructured in the upper midwest of the United States. Appl. Environ. Microbiol. 78, 8096-8101. https://doi.org/10.1128/AEM.02105-12.

Holsback, L., Lima, H.E., Vidotto, O., Silva, M.Ada, Patelli, T.H.C., Martins, F.D.C., Seixas, Mde, 2018. Cryptosporidium occurrence in ruminants from the North Pioneer mesoregion of Paraná. Brazil. Rev. Bras. Parasitol. Vet. 27, 248-253. https://doi.org/10. 1590/s1984-296120180037.

Hotchkiss, E.J., Gilray, J.A., Brennan, M.L., Christley, R.M., Morrison, L.J., Jonsson, N.N., Innes, E.A., Katzer, F., 2015. Development of a framework for genotyping bovinederived Cryptosporidium parvum, using a multilocus fragment typing tool. Parasit. Vectors 8, 500. https://doi.org/10.1186/s13071-015-1107-8.

Hunter, P.R., Gaston, M., 1988. Numerical index of the discriminatory ability of typing 
systems: an application of simpson's index of diversity. J. Clin. Microbiol. 26, 2465-2466.

Hunter, P.R., Hadfield, S.J., Wilkinson, D., Lake, I.R., Harrison, F.C.D., Chalmers, R.M., 2007. Subtypes of Cryptosporidium parvum in humans and disease risk. Emerg. Infect. Dis. 13, 82-88. https://doi.org/10.3201/eid1301.060481.

Hunter, P.R., Wilkinson, D.C., Lake, I.R., Harrison, F.C.D., Syed, Q., Hadfield, S.J., Chalmers, R.M., 2008. Microsatellite typing of Cryptosporidium parvum in isolates from a waterborne outbreak. J. Clin. Microbiol. 46, 3866-3867. https://doi.org/10. 1128/JCM.01636-08.

Khan, A., Shaik, J.S., Grigg, M.E., 2018. Genomics and molecular epidemiology of Cryptosporidium species. Acta Trop. 184, 1-14. https://doi.org/10.1016/j. actatropica.2017.10.023.

Mallon, M.E., MacLeod, A., Wastling, J.M., Smith, H., Tait, A., 2003. Multilocus genotyping of Cryptosporidium parvum Type 2: population genetics and sub-structuring. Infect. Genet. Evol. 3, 207-218. https://doi.org/10.1016/S1567-1348(03)00089-3.

Meireles, M.V., 2010. Cryptosporidium infection in Brazil : implications for veterinary medicine and public health. Rev. Bras. Parasitol. Vet. 19, 197-204. https://doi.org/ 10.1590/S1984-29612010000400002.

Meireles, M.V., De Oliveira, F.P., Teixeire, W.F., Coelho, W., Mendes, L.C.N., 2011 Molecular characterization of Cryptosporidium spp. in dairy calves from the state of São Paulo, Brazil. Parasitol. Res. 109, 949-951. https://doi.org/10.1007/s00436011-2336-1.

Mercado, R., Peña, S., Ozaki, L.S., Fredes, F., Godoy, J., 2015. Multiple Cryptosporidium parvum subtypes detected in a unique isolate of a Chilean neonatal calf with diarrhea. Parasitol. Res. 114, 1985-1988. https://doi.org/10.1007/s00436-015-4364-8.

Morrison, L.J., Mallon, M.E., Smith, H.V., MacLeod, A., Xiao, L., Tait, A., 2008. The population structure of the Cryptosporidium parvum population in Scotland: a complex picture. Infect. Genet. Evol. 8, 121-129. https://doi.org/10.1016/j.meegid.2007.10. 010.

Ngouanesavanh, T., Guyot, K., Certad, G., Fichoux, Y.Le., Chartier, C., Verdier, R.I., Cailliez, J.C., Camus, D., Dei-Cas, E., Bañuls, A.L., 2006. Cryptosporidium population genetics: evidence of clonality in isolates from France and Haiti. J. Eukaryot. Microbiol. 53, 33-36. https://doi.org/10.1111/j.1550-7408.2006.00166.x.

Paz e Silva, F., Souza, R., Joao, A.J., 2013. Identification of Cryptosporidium species and genotypes in dairy cattle in Brazil. Rev. Bras. Parasitol. Vet. 22, 22-28. https://doi. org/10.1590/s1984-29612013005000010.

Pérez-Cordón, G., Robinson, G., Nader, J., Chalmers, R.M., 2016. Discovery of new variable number tandem repeat loci in multiple Cryptosporidium parvum genomes for the surveillance and investigation of outbreaks of cryptosporidiosis. Exp. Parasitol. 169, 119-128. https://doi.org/10.1016/j.exppara.2016.08.003.

Pritchard, J.K., Stephens, M., Donnelly, P., 2000. Inference of population structure using multilocus genotype data. Genetics. 155, 945-959. https://doi.org/10.1111/j.14718286.2007.01758.x.

Pulido-Medellín, M.O., Andrade-Becerra, R.J., Iván Rodríguez-Vivas, R., Garcia-Corredor, D.J., 2014. Prevalencia y posibles factores de riesgo en la excreción de ooquistes de Cryptosporidium spp. en bovinos de Boyacá, Colombia. Rev. Mex. Ciencias Pecu. 5, 357-364. https://doi.org/10.22319/rmcp.v5i3.3981.

Quílez, J., Vergara-Castiblanco, C., Monteagudo, L., Del Cacho, E., Sánchez-Acedo, C.,
2011. Multilocus fragment typing and genetic structure of Cryptosporidium parvum isolates from diarrheic preweaned calves in Spain. Appl. Environ. Microbiol. 77 7779-7786. https://doi.org/10.1128/AEM.00751-11.

Quílez, J., Vergara-Castiblanco, C., Monteagudo, L., Del Cacho, E., Sánchez-Acedo, C., 2013. Host association of Cryptosporidium parvum populations infecting domestic ruminants in spain. Appl. Environ. Microbiol. 79, 5363-5371. https://doi.org/10. 1128/AEM.01168-13.

Ramo, A., Quílez, J., Monteagudo, L., Del Cacho, E., Sánchez-Acedo, C., 2016a. Intraspecies diversity and panmictic structure of Cryptosporidium parvum populations in cattle farms in Northern Spain. PLoS One 11, e0148811. https://doi.org/10.1371/ journal.pone.0148811.

Ramo, A., Monteagudo, L.V., Del Cacho, E., Sánchez-Acedo, C., Quílez, J., 2016b. Intraspecies genetic diversity and clonal structure of Cryptosporidium parvum in sheep farms in a confined geographical area in northeastern Spain. PLoS One 11, e0155336. https://doi.org/10.1371/journal.pone.0155336.

Robinson, G., Chalmers, R.M., 2012. Assessment of polymorphic genetic markers for multi-locus typing of Cryptosporidium parvum and Cryptosporidium hominis. Exp. Parasitol. 132, 200-215. https://doi.org/10.1016/j.exppara.2012.06.016.

Ryan, U., Fayer, R., Xiao, L., 2014. Cryptosporidium species in humans and animals: current understanding and research needs. Parasitology 14, 1667-1685. https://doi. org/10.1017/S0031182014001085.

Ryan, U., Zahedi, A., Paparini, A., 2016. Cryptosporidium in humans and animals - a one health approach to prophylaxis. Parasite Immunol. 38, 535-547. https://doi.org/10. 1111/pim.12350.

Tanriverdi, S., Widmer, G., 2006. Differential evolution of repetitive sequences in Cryptosporidium parvum and Cryptosporidium hominis. Infect. Genet. Evol. 6, 113-122. https://doi.org/10.1016/j.meegid.2005.02.002.

Tanriverdi, S., Grinberg, A., Chalmers, R.M., Hunter, P.R., Petrovic, Z., Akiyoshi, D.E., London, E., Zhang, L., Tzipori, S., Tumwine, J.K., Widmer, G., 2008. Inferences about the global population structures of Cryptosporidium parvum and Cryptosporidium hominis. Appl. Environ. Microbiol. 74, 7227-7234. https://doi.org/10.1128/AEM. 01576-08.

Thomson, S., Hamilton, C.A., Hope, J.C., Katzer, F., Mabbott, N.A., Morrison, L.J., Innes, E.A., 2017. Bovine cryptosporidiosis: impact, host-parasite interaction and control strategies. Vet. Res. 48, 42. https://doi.org/10.1186/s13567-017-0447-0.

Toledo, R.D., Martins, F.D., Ferreira, F.P., de Almeida, J.C., Ogawa, L., Dos Santos, H.L., Dos Santos, M.M., Pinheiro, F.A., Navarro, I.T., García, J.L., 2017. Cryptosporidium spp. and Giardia spp. In feces and water and the associated exposure factors on dairy farms. Plos One 12, e0175311. https://doi.org/10.1371/journal.pone.0175311.

Tomazic, M.L., Maidana, J., Dominguez, M., Uriarte, E.L., Galarza, R., Garro, C., FlorinChristensen, M., Schnittger, L., 2013. Molecular characterization of Cryptosporidium isolates from calves in Argentina. Vet. Parasitol. 198, 382-386. https://doi.org/10. 1016/j.vetpar.2013.09.022.

Xiao, L., Singh, A., Limor, J., Graczyk, T.K., Gradus, S., Lal, A., 2001. Molecular characterization of Cryptosporidium oocysts in samples of raw surface water and wastewater. Appl. Environ. Microbiol. 67, 1097-1101. https://doi.org/10.1128/AEM.67.3. 1097. 